\title{
ON AFFINELY CONNECTED MANIFOLDS WHOSE TORSION CAN BE TRANSFORMED INTO CONSTANT COMPONENTS ${ }^{1}$
}

M. PINL

Let $\bar{g}_{i k}\left(\bar{x}_{1}, \cdots, \bar{x}_{n}\right)=\bar{g}_{k i}\left(\bar{x}_{1}, \cdots, \bar{x}_{n}\right)$ be the components of the fundamental tensor of a nonsingular Riemannian space $V_{n}$ of $n$ dimensions $\left(n \geqq 2,\left|\bar{g}_{i k}\right| \neq 0\right)$ and

$$
\left\{\begin{array}{c}
i \\
k l
\end{array}\right\}^{*}, \quad \bar{R}_{k l m}^{i}
$$

the corresponding Christoffel symbols of the second kind and mixed components of curvature.

In the case of a constant metric $g_{\alpha \beta}\left(x_{1}, \cdots, x_{n}\right)$ with respect to the coordinates $x_{1}, \cdots, x_{n}$ the following relations hold for the corresponding Christoffel symbols

$$
\left\{\begin{array}{c}
\gamma \\
\alpha \beta
\end{array}\right\}
$$

and components $R_{\beta \gamma \delta}^{\alpha}$ of curvature

$$
\left\{\begin{array}{c}
\alpha \\
\beta \gamma
\end{array}\right\} \equiv 0, \quad R_{\beta \gamma \delta}^{\alpha} \equiv 0, \quad\left\{x_{1}, \cdots, x_{n}\right\}, \quad \alpha, \beta, \gamma, \delta=1,2, \cdots, n .
$$

Conversely the vanishing of Christoffel symbols

$$
\left\{\begin{array}{c}
\alpha \\
\beta \gamma
\end{array}\right\}
$$

in the identities (1) and the transformation law of Christoffel symbols require for the transformation $\bar{x} \rightarrow x$ the system of conditions

$$
\frac{\partial^{2} x_{\gamma}}{\partial \bar{x}_{i} \partial \bar{x}_{k}}=\left\{\begin{array}{l}
l \\
i k
\end{array}\right\} \frac{\partial x_{\gamma}}{\partial \bar{x}_{l}}, \quad \gamma, i, k, l=1,2, \cdots, n .
$$

Its conditions of integrability

$$
\bar{R}_{k l m}^{i} \equiv 0, \quad\left\{\bar{x}_{1}, \cdots, \bar{x}_{n}\right\}
$$

are satisfied by assumption, since the second equations of the identities (1) must be fulfilled as tensor relations in every coordinate system. Hence the well-known result [1]: the components of the funda-

Received by the editors June 10, 1959.

${ }^{1}$ Dedicated to Professor A. Brauer on the occasion of his 65 th birthday. 
mental tensor of a nonsingular Riemannian $V_{n}$ can be transformed into constants, if and only if the Riemann-Christoffel tensor of curvature identically vanishes.

In an $n$-dimensional space $A_{n}$ with asymmetric affine connection

$$
\Gamma_{\alpha \beta}^{\lambda}\left(x_{1}, \cdots, x_{n}\right) \neq \Gamma_{\beta \alpha}^{\lambda}\left(x_{1}, \cdots, x_{n}\right), \quad \lambda, \alpha, \beta=1,2, \cdots, n
$$

the differences

$$
\stackrel{\lambda}{t_{\alpha \beta}}=\Gamma_{\alpha \beta}^{\lambda}-\Gamma_{\beta \alpha}^{\lambda}=-{\stackrel{\lambda}{t_{\beta \alpha}}}^{\lambda}
$$

form the components of the simplest tensor of $A_{n}$, the so-called torsion tensor. For this tensor $t_{\alpha \beta}^{\lambda}$ we are dealing in the following with the analogous problem and put the question: under which circumstances can the components $\bar{t}_{i k}^{l}$ of the torsion of an $A_{n}$ be altogether transformed into constants $c_{\alpha \beta}^{\lambda}$, not all vanishing?

1. Associated tensors. From the $n^{2}(n-1) / 2$ components of the torsion tensor $t_{\alpha \beta}^{\lambda}$ of an affinely connected $A_{n}$ we get by contraction with respect to $\lambda=\alpha$ the associated torsion vector [2]

$$
t_{\beta}=\stackrel{\lambda}{t_{\lambda \beta}}
$$

and by inner multiplication ("Überschiebung") the associated symmetric covariant quadratic torsion tensor [2, p. 191]

$$
t_{\alpha \beta}=t_{\alpha \mu}^{\lambda} t_{\beta \lambda}^{\mu}=t_{\beta \alpha}, \quad \alpha, \beta, \lambda, \mu=1,2, \cdots, n .
$$

For $n=2$

$$
\frac{n^{2}(n-1)}{2}=2, \quad t_{12}^{1}=t_{2}, \quad t_{21}^{2}=t_{1}
$$

hold. In this case the torsion tensor $t_{\alpha \beta}^{\lambda}$ is already given by the components $t_{\beta}$ of the torsion vector (2); the quadratic torsion tensor (3) is a singular one, $\left|t_{\alpha \beta}\right|=0$, but its rank is 1 , if the case of vanishing torsion, $t_{\alpha \beta}^{\lambda}=0$, should be excluded. Nevertheless the matrix

$$
\left\|t_{23}^{\lambda}, t_{31}^{\lambda}, t_{12}^{\lambda}\right\|, \quad \lambda=1,2,3
$$

has at least the rank 1 in the case $n=3$, even if the relations $t_{\alpha \beta}=0$, $t_{\beta}=0$ are allowed [2, pp. 196-197].

In the general case, $\left|t_{\alpha \beta}\right| \neq 0$, as is well known, the contravariant tensor $t^{\alpha \beta}$ can be associated with the covariant tensor $t_{\alpha \beta}$. 
2. $n=2$. For $n=2$ the binary Pfaffian

$$
\omega=t_{1} d x_{1}+t_{2} d x_{2}
$$

is given by (4). Let the coefficients $t_{1}, t_{2}$ be constants, $t_{\beta}=c_{\beta}$. Then the bilinear covariant $\omega^{\prime}$ of the form (5) vanishes identically and the class of $\omega$ is 1 . Conversely $\omega$ can be reduced to the canonical form $\bar{\omega}=1 \cdot d \bar{x}_{1}+0 \cdot d \bar{x}_{2}$, if $\omega^{\prime} \equiv 0$. Thus we get:

(I) The torsion $t_{\alpha \beta}^{\lambda}$ of an affinely connected surface $A_{2}$ can be transformed into constant components not all zero, precisely if its torsion vector $t_{\beta}$ is a gradient vector.

If a vector $\xi^{\alpha}$ along a curve $x_{i}(s)$ in $A_{2}$ is autoparallel for all possible choices of the torsion, then $\xi^{\alpha}$ is necessarily the tangent vector of an autoparallel curve [3]. The differential condition that such a vector (in $A_{n}$ ) must satisfy is $t_{\alpha \beta}^{i} \xi^{\alpha} x^{\prime \beta}=0$, as one sees by writing the equations of parallelism in the form $\left(\xi^{i}\right)^{\prime}+\Gamma_{(\alpha \beta)}^{i} \xi^{\alpha} x^{\prime \beta}+t_{\alpha \beta}^{i} \xi^{\alpha} x^{\prime \beta} / 2=0$. For $n=2$ this reduces to $t_{12}^{\prime}\left(\xi^{1} x^{\prime 2}-x^{\prime 2} \xi^{1}\right)=0$, for all nonzero $t_{12}^{t}$, and therefore $\left(\xi^{1}, \xi^{2}\right)=k\left(x^{\prime 1}, x^{\prime 2}\right)$.

3. $n>2,\left|t_{\alpha \beta}\right| \neq 0$. In the case $n>2,\left|t_{\alpha \beta}\right| \neq 0$, a nonsingular Riemannian space $V_{n}$ of $n$ dimensions is associated to the affinely connected space $A_{n}$ with the torsion $t_{\alpha \beta}^{\lambda}$. The fundamental tensor of this $V_{n}$ is $t_{\alpha \beta}$. We can use the brackets of Christoffel

$$
\left\{\begin{array}{c}
\lambda \\
\alpha \beta
\end{array}\right\}, \quad[\lambda, \alpha \beta]
$$

of the tensor $t_{\alpha \beta}$, to define covariant derivatives $t_{\alpha \beta(\gamma)}^{\lambda}$ of the torsion tensor $t_{\alpha \beta}^{\lambda}$. We get

$$
\left\{\begin{array}{c}
\lambda \\
\alpha \beta
\end{array}\right\}=t^{\lambda \zeta}[\zeta, \alpha \beta]=\frac{1}{2} t^{\lambda \zeta}\left(\frac{\partial t_{\alpha \zeta}}{\partial x_{\beta}}+\frac{\partial t_{\beta \zeta}}{\partial x_{\alpha}}-\frac{\partial t_{\alpha \beta}}{\partial x_{\zeta}}\right)=\left\{\begin{array}{c}
\lambda \\
\beta \alpha
\end{array}\right\}
$$

and

$$
\hat{t}_{\alpha \beta(\gamma)}^{\lambda}=\frac{\partial t_{\alpha \beta}}{\partial x_{\gamma}}+\left\{\begin{array}{c}
\lambda \\
\alpha \zeta
\end{array}\right\} t_{\alpha \beta}^{\zeta}-\left\{\begin{array}{c}
\zeta \\
\alpha \gamma
\end{array}\right\} \hat{t}_{\zeta \beta}^{\lambda}-\hat{t}_{\alpha \zeta}^{\lambda}\left\{\begin{array}{c}
\zeta \\
\beta \gamma
\end{array}\right\} .
$$

If the components $t_{\alpha \beta}^{\lambda}$ can be reduced to the constant values $c_{\alpha \beta}^{\lambda}$ in the coordinate system $x_{1}, \cdots, x_{n}$, the same is true with respect to (3) for the components $t_{\alpha \beta}$ of the associated quadratic torsion tensor. Therefore the Christoffel brackets

$$
\left\{\begin{array}{c}
\lambda \\
\alpha \beta
\end{array}\right\}
$$


the corresponding Riemann-Christoffel tensor of curvature

$$
R_{\alpha \beta \gamma}^{\lambda}=\frac{\partial\left\{\begin{array}{c}
\lambda \\
\alpha \gamma
\end{array}\right\}}{\partial x_{\beta}}-\frac{\partial\left\{\begin{array}{c}
\lambda \\
\alpha \beta
\end{array}\right\}}{\partial x_{\gamma}}+\left\{\begin{array}{c}
\sigma \\
\alpha \gamma
\end{array}\right\}\left\{\begin{array}{c}
\lambda \\
\sigma \beta
\end{array}\right\}-\left\{\begin{array}{c}
\sigma \\
\alpha \beta
\end{array}\right\}\left\{\begin{array}{c}
\lambda \\
\sigma \gamma
\end{array}\right\}
$$

and the tensor $t_{\alpha \beta}^{\lambda}(\gamma)$ simultaneously are zero, if the derivatives $\partial t_{\alpha \beta}^{\lambda} / \partial x_{\gamma}$ vanish. Vice versa: if the tensor $R_{\alpha \beta \gamma}^{\lambda}$ vanishes in any special system of coordinates, the same is true in all coordinate systems; then special coordinate systems $x_{1}, \cdots, x_{n}$ exist, for which, following (6),

$$
\left\{\begin{array}{c}
\lambda \\
\alpha \beta
\end{array}\right\} \equiv 0, \quad\left\{x_{1}, \cdots, x_{n}\right\}
$$

In such coordinate systems, following (7), the relations

$$
\stackrel{\partial t_{\alpha \beta(\gamma)}^{\lambda}}{\lambda}=\frac{\partial t_{\alpha \beta}}{\partial x_{\gamma}}
$$

hold. Thus we get

$$
\frac{\partial t_{\alpha \beta}^{\lambda}}{\partial x_{\gamma}}=0 \quad \text { or } \quad \hat{t}_{\alpha \beta}^{\lambda}=\stackrel{\lambda}{c_{\alpha \beta}}
$$

if $R_{\alpha \beta \gamma}^{\lambda} \equiv 0$ and $t_{\alpha \beta(\gamma)}^{\lambda} \equiv 0$. Hence,

(II) The torsion $t_{\alpha \beta}^{\lambda}$ of a $n$-dimensional affinely connected space $A_{n}$ $(n \geqq 3)$ with

$$
\left|t_{\alpha \beta}\right|=\left|t_{\alpha \mu}^{\lambda} t_{\beta \lambda}^{\mu}\right| \neq 0
$$

can be transformed into constant components $c_{\alpha \beta}^{\lambda}$ not all zero, precisely if the Riemann space $V_{n}$ associated with $A_{n}$ is a euclidean space $E_{n}$ and the tensor $t_{\alpha \beta(\gamma)}^{\lambda}$, as given by (7), vanishes.

Suppose $n \geqq 3$. $\left|c_{\alpha \beta}\right| \neq 0$; if arbitrary autoparallel vectors along an autoparallel curve of $A_{n}$ remain autoparallel under all possible changes of the torsion, then the paths of the connection are integrals of the Pfaffian system [3, p. 559]

$$
\stackrel{\lambda}{c_{\alpha 1}} d x_{1}+\cdots+\stackrel{\lambda}{c_{\alpha m}} d x_{n}=0, \quad \alpha, \lambda=1,2, \cdots, n .
$$

4. $n=3$. Examples. For $n=3$ we get $n^{2}(n-1) / 2=9$ components $t_{\alpha \beta}^{\lambda}, n(n+1) / 2=6$ components $t_{\alpha \beta}$ and 3 components $t_{\beta}$. After an euclidean metric

$$
t_{11}=1, t_{12}=0, t_{13}=-1, t_{22}=1, t_{23}=0, t_{33}=1
$$


has been chosen, we put

$$
\begin{aligned}
& t_{12}^{1}=y_{1}, \stackrel{1}{t_{13}}=y_{2}, \stackrel{1}{t_{23}}=y_{3} ; \stackrel{2}{t_{12}}=y_{4}, \stackrel{2}{t_{13}}=y_{5}, \stackrel{2}{t_{23}}=y_{6} ; \\
& \stackrel{3}{t_{12}}=y_{7}, \stackrel{3}{t_{13}}=y_{8}, \stackrel{3}{t_{23}}=y_{9} \text {. }
\end{aligned}
$$

Then, following (3), the quadratic system of 6 equations for 9 unknowns $y_{1}, y_{2}, \cdots, y_{9}$ becomes:

$$
\begin{aligned}
t_{11} & =y_{4}^{2}+2 y_{5} y_{7}+y_{8}^{2}=1, \quad t_{12}=-y_{1} y_{4}-y_{7}\left(y_{2}-y_{6}\right)+y_{8} y_{9}=0, \\
\text { (10) } t_{22} & =y_{1}^{2}-2 y_{3} y_{7}+y_{9}^{2}=1,-t_{13}=y_{1} y_{5}+y_{2} y_{8}+y_{4} y_{6}+y_{5} y_{9}=1, \\
t_{33} & =y_{2}^{2}+2 y_{3} y_{5}+y_{6}^{2}=1, \quad t_{23}=y_{1} y_{2}+y_{3}\left(y_{4}-y_{8}\right)-y_{6} y_{9}=0 .
\end{aligned}
$$

Let $f\left(x_{1}, x_{2}, x_{3}\right)$ be an arbitrary (differentiable) function. We put:

$$
\begin{gathered}
y_{1}=y_{2}=y_{4}=y_{6}=y_{8}=y_{9}=\frac{1}{+2^{1 / 2}}, \\
y_{3}=y_{5}=0, \quad y_{7}=f\left(x_{1}, x_{2}, x_{3}\right) .
\end{gathered}
$$

The values (11) satisfy the system (10) for arbitrary functions $f$, i.e. for arbitrarily chosen components $t_{12}^{3}$.

Now we denote by $\Gamma_{(\alpha \beta)}^{\lambda}\left(x_{1}, \cdots, x_{n}\right)$ an arbitrary symmetric linear connection. Then the three dimensional affinely connected spaces $A_{3}$, which correspond to the torsion (11) have the asymmetric connection [2, p. 191]:

$$
\begin{array}{rrr}
\Gamma_{12}^{1}=\Gamma_{(12)}^{1}+\frac{1}{2(2)^{1 / 2}}, & \Gamma_{13}^{1}=\Gamma_{(13)}^{1}+\frac{1}{2(2)^{1 / 2}}, & \Gamma_{23}^{1}=\Gamma_{(23)}^{1}, \\
\Gamma_{12}^{2}=\Gamma_{(12)}^{2}+\frac{1}{2(2)^{1 / 2}}, & \Gamma_{13}^{2}=\Gamma_{(13)}^{2}, \quad \Gamma_{23}^{2}=\Gamma_{(23)}^{2}-\frac{1}{2(2)^{1 / 2}}, \\
\Gamma_{12}^{3}=\Gamma_{(12)}^{3}+\frac{1}{2} f\left(x_{1}, x_{2}, x_{3}\right), & \Gamma_{13}^{3}=\Gamma_{(13)}^{3}-\frac{1}{2(2)^{1 / 2}}, & \Gamma_{23}^{3}=\Gamma_{(23)}^{3}-\frac{1}{2(2)^{1 / 2}}, \\
& & \left(\Gamma_{\alpha \beta}^{\lambda}=\Gamma_{(\alpha \beta)}^{\lambda}+\frac{1}{2} \underset{t \alpha \beta}{\lambda}\right) .
\end{array}
$$

Since the components (9) of the euclidean metric have been chosen as constants, the corresponding Christoffel brackets vanish and the covariant derivatives $t_{\alpha \beta(\gamma)}^{\lambda}$ of the torsion tensor are reduced, following (7), to the derivatives $\partial t_{\alpha \beta}^{\lambda} / \partial x_{\gamma}$. But the derivatives $\partial t_{12}^{3} / \partial x_{\gamma}$ can only be zero, if $f=$ const. Thus we may say:

(III) The conditions $R_{\alpha \beta \gamma}^{\lambda}=0$ and $t_{\alpha \beta(\gamma)}^{\lambda}=0$ of Theorem (II) are independent conditions.

The conditions for $t_{\alpha \beta}^{\lambda}=c_{\alpha \beta}^{\lambda}$, developed in the foregoing in the case 
$n>2$, require that nonsingular Riemann spaces can be associated with the torsion tensor $\left(\left|t_{\alpha \beta}\right| \neq 0\right)$. Regarding singular Riemann spaces we are especially interested in "reducible" singular Riemann spaces whose matrix $\left\|t_{\alpha \beta}\right\|$ can be reduced to the canonical form

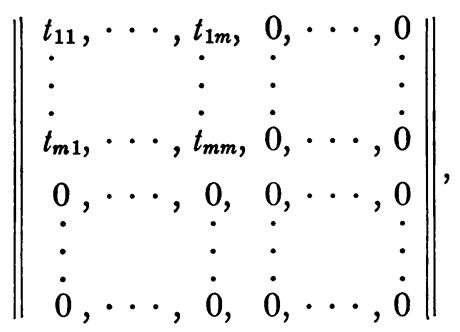

if $d=n-m$ is the defect of the matrix $\left\|t_{\alpha \beta}\right\|$. The theory of linear connections in such singular Riemann spaces as developed by $A$. $P$. Norden [5] $(d=1)$ and C. Jankiewicz [6] $(d>1)$ also seems to be interesting in regard to the problem of constant components $t_{\alpha \beta}^{\lambda}=c_{\alpha \beta}^{\lambda}$ in such singular cases. It may be examined later on [7].

\section{REFERENCES}

1. R. Weitzenböck, Invariantentheorie, Groningen, 1923, p. 340.

2. G. Vranceanu, Leçons de geometrie differentielle I, Bucarest, 1957, pp. 190-197.

3. M. Pinl, Geodesic coordinates and rest systems for general linear connections, Duke Math. J. vol. 18 (2) (1951) pp. 557-562.

4. L. Pf. Eisenhart, Riemannian geometry, Princeton, 1949, pp. 26-31.

5. A. P. Norden, Prostranstva afinot sviaznosti, Dokl. Akad. Nauk SSSR vol. 50 (1945) p. 37; Moscou, 1950, §\$76-77.

6. C. Jankiewicz, Sur les espaces Riemanniens dégénerês, Bull. Acad. Polon. Sci. Cl. III, vol. 2, no. 7 (1954) pp. 301-304.

7. M. Pinl, Über lineare Übertragungen mit fallweise konstanter Torsion, J. Reine Angew. Math., Festband für W. Krull, 1960.

Mathematisches Institut der Universitat KölN, Köln, Germany 